${ }^{1}$ Fundación Oftalmológica de Los Andes, Santiago, Chile. ${ }^{2}$ Hospital Dr. Sótero del Río, Santiago, Chile.

anterno Medicina Universidad de Los Andes, Santiago, Chile.

bInterna Medicina Pontificia

Universidad Católica de Chile, Santiago, Chile.

Trabajo no recibió financiamiento. Los autores declaran no tener conflictos de interés.

Recibido el 19 de abril de 2018 Aceptado el 14 de mayo de 2019

Correspondencia a: Manuel Gallegos Universidad de Los Andes, Santiago, Chile. magallegos@miuandes.cl

\section{Repercusiones oculares del Síndrome de Alport: A propósito de dos casos}

\author{
ALEX JONES ${ }^{1}$, MANUEL GALLEGOS ${ }^{a}$, IGNACIO DÍAZ², \\ MAGDALENA REYES ${ }^{\mathrm{b}}$, SERGIO ZACHARIAS ${ }^{1}$
}

\section{Alport syndrome. Report of two cases}

Alport syndrome is an inherited progressive form of glomerular disease that is often associated with sensorineural hearing loss and ocular abnormalities. We report two men with Alport syndrome. Both had chronic kidney disease and consulted for long-term loss of visual acuity. One had auditory abnormalities. On the ophthalmological examination, both had anterior lenticonus and one had dot or fleck retinopathy. Those findings are described in up to 50\% and 70\% of men with X-linked Alport syndrome, respectively. Both patients had a family history of Alport syndrome or suggestive signs and symptoms.

(Rev Med Chile 2019; 147: 522-526)

Key words: Lens Diseases; Nephritis, Hereditary.
$\mathrm{E}$ 1 Síndrome de Alport (SA) corresponde a una enfermedad hereditaria caracterizada por hematuria, falla renal progresiva, pérdida de la audición y anormalidades oculares secundarias a mutaciones en los genes que codifican para el colágeno tipo IV, elemento estructural de la membrana basal ${ }^{1}$.

Además, diversas isoformas del colágeno IV $(\alpha 3, \alpha 4$ o $\alpha 5)$ se ubican en membranas y órganos diferentes ${ }^{3}$, lo que explica el compromiso tanto de riñón, ojos y cóclea en un paciente con SA (Tabla 1).

Su prevalencia es variable, siendo ésta de $1 / 10.000^{1}$ hasta $1 / 50.000$ en la población general, con predominio y mayor severidad en hombres ${ }^{3}$. Por otra parte, el SA se encuentra en alrededor del $3 \%$ de los niños con enfermedad renal terminal ${ }^{3}$.

$\mathrm{Su}$ forma de presentación más frecuente es ligada al cromosoma $\mathrm{X}$, la cual se debe a mutación del gen COL4A5 ${ }^{5}$. Ésta variedad corresponde a 80-85\% de los casos, afectando principalmente a varones; las mujeres rara vez presentan la enfermedad ya que son mayoritariamente portadoras ${ }^{6}$. Las formas autosómica recesiva y autosómica dominante representan $15 \%$ y $5 \%$ de los casos respectivamente y ambas ocurren por mutaciones en los genes COL4A3 o COL4A $4^{4}$.
La afección ocular se presenta en 15-30\% de los casos $^{2}$, es bastante específica y en ocasiones patognomónica lo que obliga a plantear el diagnóstico. Sin embargo, su aparición no es temprana, llegando a evidenciarse cierto grado de nefritis crónica y sordera neurosensorial en todos los pacientes con lenticono anterior los cuales, a lo largo de los próximos 30 años, habrán progresado a enfermedad renal terminal y sordera ${ }^{13}$.

El presente artículo tiene por objetivo describir dos casos de SA atendidos en Fundación Oftalmológica Los Andes y sus hallazgos oftalmológicos. Ambos con consentimiento informado de por medio.

\section{Caso 1}

Paciente masculino de 28 años con antecedentes de enfermedad renal crónica, SA diagnosticado hace 8 años y en tratamiento con hemodiálisis trisemanal hace 7 años. Sin antecedentes quirúrgicos relevantes. Tratamiento farmacológico con metil-dopa, nitrendipino, atenolol, losartán, ácido fólico y aspirina. Antecedente familiar de hermano en hemodiálisis con diagnóstico de SA. Consulta por disminución de 
Tabla 1. Distribución cadenas alfa 3, 4 y 5 en el organismo

\begin{tabular}{|lll|}
\hline Riñón & Ojo & Cóclea \\
\hline Membrana basal glomerular & Cápsula del cristalino & Surco espiral interno y externo \\
\hline Cápsula de Bowman & Membrana de Descemet & Limbo espiral \\
\hline Membrana basal tubular distal & Membrana de Bruch & Prominencia espiral \\
\hline & Membrana limitante interna & Membrana basilar \\
\hline
\end{tabular}

Fuente (3).

la agudeza visual (AV) bilateral de larga data sin hipoacusia asociada.

$\mathrm{Al}$ examen físico tonometría ocular ojo derecho (OD) $8 \mathrm{mmHg}$ e izquierdo (OI) $9 \mathrm{mmHg}$, AV mejor corregida ojo derecho $0,12 \mathrm{P}$ e izquierdo $0,12+1$. Al Biomicroscopio (BMC) se identifica lenticono anterior bilateral, catarata polar posterior bilateral. Al fondo de ojo (FO) se aprecia losanjo macular con depósitos druseiformes periféricos poco definidos, sin otros hallazgos en periferia (Figura 1). Se entiende por losanjo al reflejo opaco debido al contraste entre la mácula y el puntillado perifoveal. A la tomografía de coherencia óptica (OCT) que evidencia mácula con leve atrofia. Fue sometido posteriormente a facoeresis con lente intraocular en ambos ojos, mostrando importante mejoría en su AV, alcanzando 0,8 en ambos ojos. Actualmente estable con controles anuales.

\section{Caso 2}

Paciente masculino de 37 años con antecedentes enfermedad renal crónica tratada con doble transplante renal, SA diagnosticado hace 21 años e hipoacusia. Operado de catarata ojo izquierdo. Antecedentes familiares de madre y 2 hermanos con SA. Consulta por disminución de AV de larga data asociada al inicio de tacrolimus.

$\mathrm{Al}$ examen físico tonometría ocular bilateral de $13 \mathrm{mmHg}$, AV mejor corregida ojo derecho 0,4 e izquierdo 0,7 ; sin defecto pupilar aferente relativo (DPAR). Al BMC, en ojo derecho se observa catarata y lenticono anterior (Figura 2); en ojo izquierdo, pseudofaquia con leve opacidad en capsula posterior. El FO reveló drusas pequeñas en zona macular, fibrosis intraretinal que dibuja los márgenes de la mácula, retina periférica y papila del nervio óptico normales (Figura 2). Al OCT se

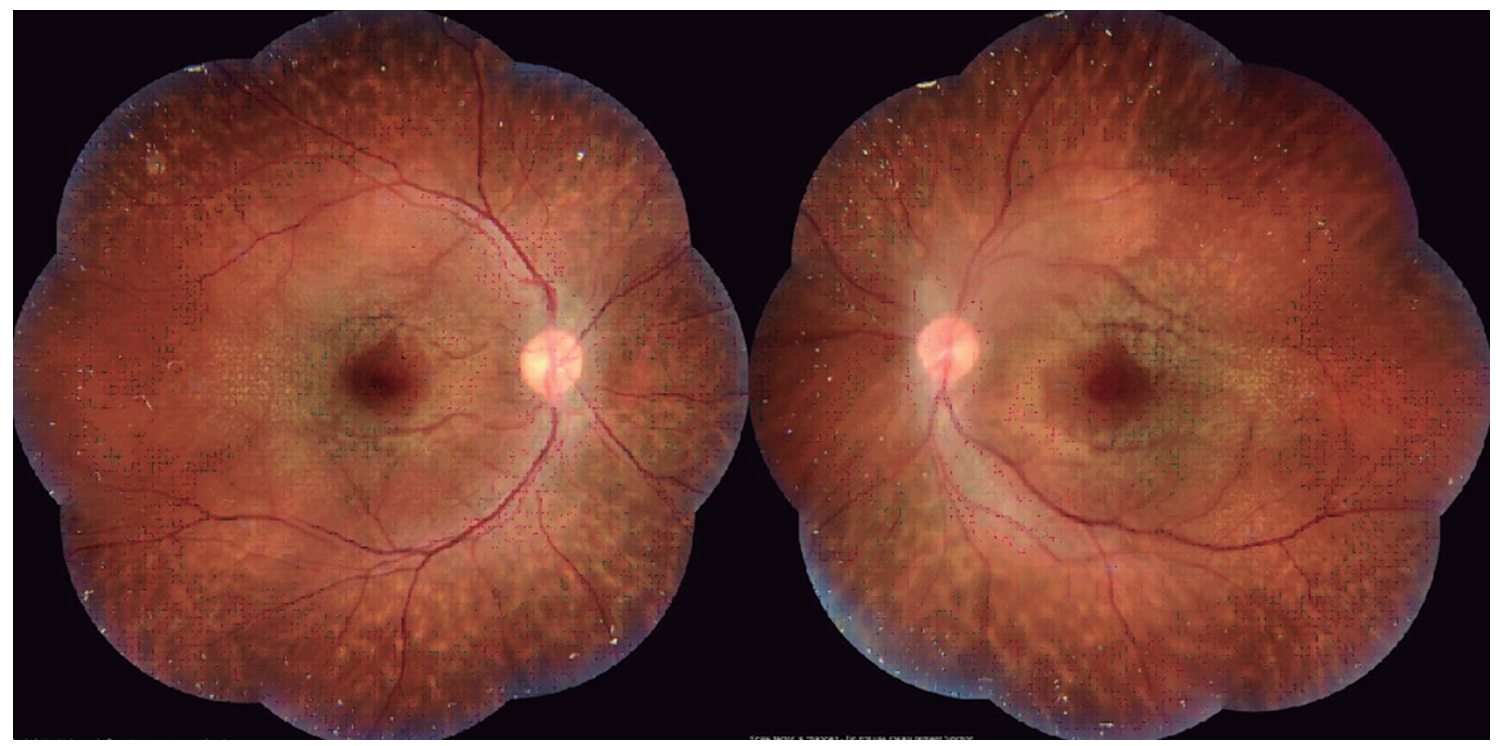

Figura 1. FO ojo derecho e izquierdo paciente número 1. 


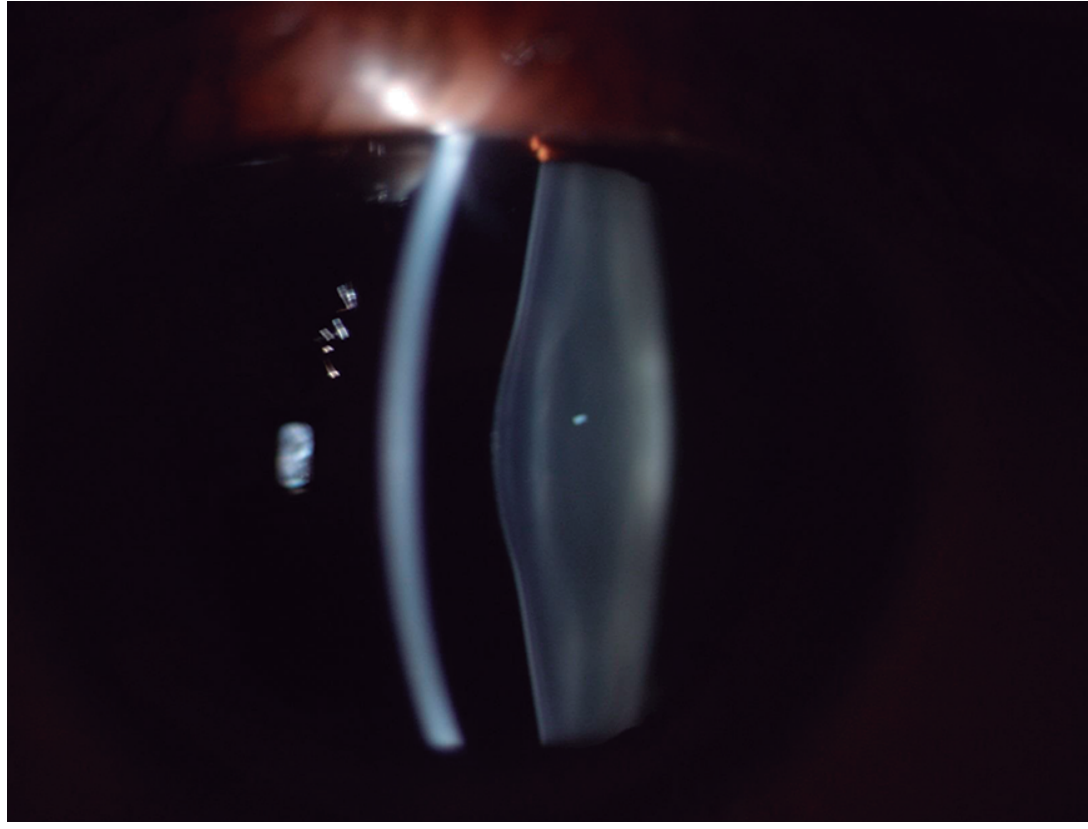

Figura 2. Lenticono anterior al BMC de paciente número 2.

evidencia mácula con leve atrofia foveolar de todas sus capas, determinada mediante comparación con medidas extraídas de una población general de referencia. ERGmf realizado con adecuada cooperación del paciente, indicó mínima alteración del anillo central y disminución leve del resto de los anillos en ambos ojos, con latencias normales $\mathrm{y}$ amplitud disminuida.

\section{Discusión}

Se han presentado dos casos de SA con manifestaciones oculares que, como se describe en la literatura, siguieron al compromiso renal. Los hallazgos oculares a pesar de no ser sintomáticos, coincidiendo de igual forma con lo descrito al respecto, son de gran valor diagnóstico pudiendo prácticamente confirmar un $\mathrm{SA}^{1}$.

Las anomalías oculares, si bien son menos sensibles que las auditivas, son altamente específicas ya que éstas últimas pudieran ocurrir en otras patologías renales hereditarias ${ }^{1}$. Dentro de las estructuras oculares más frecuentes comprometidas se encuentran el cristalino, retina y córnea $^{2}$, pudiendo apreciarse opacidades corneales, lenticono anterior, catarata, adelgazamiento de la retina temporal y retinopatía punto-mancha ${ }^{1}$.
Dentro de la amplia gama de signos al examen físico, la retinopatía punto-mancha y el lenticono anterior cobran especial importancia. La primera es la lesión retinal más frecuente ${ }^{12}$, describiéndose hasta en el 70\% de los casos masculinos de SA ligados al cromosoma $\mathrm{X}$ y esencialmente no altera la agudeza visual, aunque algunas pruebas de función retinal demuestren anormalidades menores $^{1}$. El lenticono anterior, por otro lado, se observa hasta en el 50\% de los casos ligados al cromosoma $\mathrm{X}$ y se asocia a compromiso renal temprano. Además, dado la alta especificidad de éste hallazgo, su presencia prácticamente confirma el diagnóstico de SA. En nuestros pacientes se objetivó lenticono anterior y retinopatía en ambos pacientes, observándose en el primer caso el losanjo característico. Al estudio mediante OCT de mácula se evidenciaron mínimas alteraciones que además, como se demostró en el primer caso, poco contribuían a la pérdida de $\mathrm{AV}$, coincidiendo con el resto de la literatura sobre el tema.

Otro aspecto relevante a dar a conocer es la diferencia entre el compromiso ocular y el nefrológico observado en estos pacientes. El primero suele ser progresivo y estable en el tiempo, en cambio el daño renal se caracteriza por ser rápido, precoz y determinante en la morbimortalidad posterior. Ambos pacientes fueron derivados a la institución 
para resolución de su patología ocular, por lo que no fue posible contar con un registro de las agudezas visuales previas al momento de consulta para objetivar la lenta progresión del daño.

Cabe destacar en el segundo caso el uso de tacrolimus concomitante, ya que dicho medicamento se ha asociado por sí mismo a una disminución en la AV. En diferentes artículos ${ }^{15,16}$ se ha descrito el compromiso de nervio óptico en forma de neuritis isquémica como mecanismo por el cual generaría los síntomas. Ante este escenario cobran importancia la ausencia de DPAR y la visualización de una papila normal al FO para hacer poco probable que el medicamento sea el responsable.

Consideramos relevante destacar que el valor de este reporte de casos no está en la presentación de nuevos hallazgos en el contexto de SA, sino en, a partir de la descripción de dos casos, realizar una adecuada revisión de los signos clásicos o más frecuentes en esta patología.

Frente a un paciente joven con función renal alterada e hipoacusia neurosensorial, es importante un examen oftalmológico completo a la brevedad que incluya lámpara de hendidura, fotografía de retina y OCT ya que son pruebas no invasivas y aceptables para el paciente ${ }^{1}$. Si se tratara de un niño o adolescente, es menos frecuente la presencia de manifestaciones oculares, por lo que se debe examinar a su madre, especialmente en busca de retinopatía periférica ${ }^{1}$. La razón está en la rápida progresión y morbilidad asociada al daño renal en comparación con el compromiso ocular, el cual como se ha mencionado a lo largo del trabajo, rara vez generan síntomas en el paciente. Debido a esto el oftalmólogo tiene un rol en la pesquisa precoz de las lesiones oculares para el diagnóstico consecuente de SA con el objetivo de lograr una intervención en la función renal residual del paciente u otros miembros familiares con potencial riesgo, especialmente de sexo masculino. Para lograr una evaluación precoz, el médico no oftalmólogo debe mantener un alto índice de sospecha y derivar ante la presencia de compromiso renal $y$ auditivo en un paciente joven con antecedentes familiares presentes.

Las guías actuales para el manejo de SA recomiendan que el diagnóstico sea efectuado con estudio genético ${ }^{14}$ consistente en ampliación completa por PCR de genes de colágeno. Ante dicha propuesta, el hallazgo de cualquier signo of- talmológico sugerente provee al tratante evidencia adicional para la solicitud del estudio ${ }^{1}$.

\section{Referencias}

1. Savige J, Sheth S, Leys A, Nicholson A, Mack H, Colville D. Ocular Features in Alport Syndrome: Pathogenesis and Clinical Significance. Clin J Am Soc Nephrol 2015; 10 (4): 703-9.

2. Govan J. Ocular manifestations of Alport's syndrome: a hereditary disorder of basement membranes? Br J Ophthalmol 1983; 67 (8): 493-503. Available from: https:// www.ncbi.nlm.nih.gov/pubmed/6871140 [citado el 12 de abril de 2017].

3. Kashtan C. Alport Syndrome: An Inherited Disorder of Renal, Ocular, and Cochlear Basement Membranes. Medicine 1999; 78 (5): 338-60. Available from: https:// www.ncbi.nlm.nih.gov/pubmed/10499074 [citado el 12 de abril de 2017].

4. Longo I, Porcedda P, Mari F, Giachino D, Meloni I, Deplano C, et al. COL4A3/COL4A4 mutations: From familial hematuria to autosomal-dominant or recessive Alport syndrome. Kidney Int 2002; 61 (6): 1947-56. Available from: https://www.ncbi.nlm.nih.gov/pubmed/12028435 [citado el 12 de abril de 2017].

5. Feingold J, Bois E, Chompert A, Broyer M, Gubler M, Grünfeld J. Genetic heterogeneity of Alport syndrome. Kidney Int 1985; 27 (4): 672-7. Available from: https:// www.ncbi.nlm.nih.gov/pubmed/4010153 [citado el 12 de abril de 2017].

6. Barker D, Hostikka S, Zhou J, Chow L, Oliphant A, Gerken S, et al. Identification of mutations in the COL4A5 collagen gene in Alport syndrome. Science 1990; 248 (4960): 1224-7. Available from: https://www.ncbi. nlm.nih.gov/pubmed/2349482 [citado el 12 de abril de 2017].

7. Seymenoğlu G, Baser E. Ocular manifestations and surgical results in patients with Alport syndrome. J Cataract Refract Surg 2009; 35 (7): 1302-6. Available from: http:// www.jcrsjournal.org/article/S0886-3350(09)00385-X/ abstract [citado el 12 de abril de 2017].

8. Fawzi A, Lee N, Eliott D, Song J, Stewart J. Retinal findings in patients with Alport Syndrome: expanding the clinical spectrum. Br J Ophthalmol 2009; 93 (12): 1606-11. Available from: https://www.ncbi.nlm.nih.gov/ pubmed/19635720 [citado el 12 de abril de 2017].

9. Ahmed F, Kamae K, Jones D, DeAngelis M, Hageman G, Gregory M, et al. Temporal Macular Thinning Associated With X-Linked Alport Syndrome. JAMA Ophthalmol 2013; 131 (6): 777-82. Available from: https:// 
www.ncbi.nlm.nih.gov/pubmed/23572034 [citado el 12 de abril de 2017].

10. Thomas A, Baynham J, Flaxel C. Macular holes, vitelliform lesions, and midperipheral retinoschisis in Alport syndrome. Retin Cases Brief Rep 2016; 10 (2): 109-11. Available from: https://www.ncbi.nlm.nih.gov/pubmed/26200386 [citado el 12 de abril de 2017].

11. Streeten B, Robinson M, Wallace R, Jones D. Lens Capsule Abnormalities in Alport's Syndrome. Arch Ophthalmol 1987; 105 (12): 1693-7. Available from: https:// www.ncbi.nlm.nih.gov/pubmed/3689194 [citado el 12 de abril de 2017].

12. Sabates R, Krachmer J, Weingeist T. Ocular Findings in Alport's Syndrome. Ophthalmologica 1983; 186 (4): 204-10. Available from: https://www.ncbi.nlm.nih.gov/ pubmed/6602962 [citado el 12 de abril de 2017].

13. Nielsen CE. Lenticonus anterior and Alport's syndrome. Acta Ophthalmologica 2009; 56 (4): 518-30. Available from: https://www.ncbi.nlm.nih.gov/pubmed/735766 [citado el 12 de abril de 2017].

14. Savige J, Gregory M, Gross O, Kashtan C, Ding J, Flinter F. Expert Guidelines for the Management of Alport Syndrome and Thin Basement Membrane Nephropathy. J Am Soc Nephrol 2013; 24 (3): 364-75. Available from: https://www.ncbi.nlm.nih.gov/pubmed/23349312 [citado el 12 de abril de 2017].

15. Rasool N, Boudreault K, Lessell S, Prasad S, Cestari DM. Tacrolimus Optic Neuropathy. J Neuroophthalmol. 2018; 38 (2): 160-6. doi:10.1097/ WNO.0000000000000635. PubMed PMID: 29420328.

16. Gupta M, Bansal R, Beke N, Gupta A. Tacrolimus-induced unilateral ischaemicoptic neuropathy in a non-transplant patient. BMJ Case Rep 2012; 2012. pii: bcr2012006718. doi: 10.1136/bcr-2012-006718. PubMed PMID: 22914240; PubMedCentral PMCID: PMC4544422. 